Proyecciones

Vol. 19, No 2, pp. 105-112, August 2000

Universidad Católica del Norte

Antofagasta - Chile

\title{
A VARIATIONAL INEQUALITY RELATED TO AN ELLIPTIC OPERATOR
}

\author{
A. WANDERLEY \\ Universidade do Estado do Río de Janeiro, Brasil \\ and \\ N. RIBEIRO \\ Universidade Federal do Río de Janeiro, Brasil
}

$$
\begin{aligned}
& \text { Abstract } \\
& \text { It is considered the non-linear operator } \\
& \quad A(u)=-\sum_{i=1}^{n} \frac{\partial}{\partial x_{i}}\left(\left|\frac{\partial u}{\partial x_{i}}\right|^{p-2} \frac{\partial u}{\partial x_{i}}\right), \quad p>2,
\end{aligned}
$$

and a variational inequality associated to the operator

$$
A(u)+g(x, u)
$$

with $g$ satisfying some conditions.

Key words and phrases : Non-linear operator, variational inequality, Sobolev spaces

AMS Subject Classification : Primary: 35A15; Secondary: $35 B 99$. 
We will be considering the elliptic operator:

$$
A(u)=-\sum_{i=1}^{n} \frac{\partial}{\partial x_{i}}\left({\frac{\partial u}{\partial x_{i}}}^{p-2} \frac{\partial u}{\partial x_{i}}\right), \quad p>2
$$

and a variational inequality associated to the operator

$$
A(u)+g(x, u)
$$

with $g$ satisfying some conditions, on a not necessarily bounded domain $\Omega \subset \mathbf{R}^{n}$.

We will assume that $g(x, u)$ satisfies the following hypothesis:

(a) $g(x, r)$ is measurable, in $x$, on $\Omega$, for a fixed $r \in \mathbf{R}$; it is continuous in $r$, for each $x$, fixed. For each $x \in \Omega, g(x, 0)=0$ and for all $r \in \mathbf{R}, x \in \Omega, g(x, r) r \geq 0$;

(b) $g(x, r)$ is a non-decreasing function in $r$, on $\mathbf{R}$. For each fixed $r, g_{r}(x)=g(x, r)$ is a $L^{1}(\Omega)$-function.

Let us remaind that, under $(\mathrm{b})$, if

$$
G(x, r)=\int_{0}^{r} g(x, s) d s,
$$

$G$ is continuous, convex, in $r$, for all $x$ and $r$, with $G(x, 0)=0$.

Moreover,

$$
G^{\prime}(x, r)=g(x, r) .
$$

In what follows we will use the notation as in [3].

Our goal is to prove the following theorem, where $\Omega \subset \mathbf{R}^{n}$ is an open subset and $A(u)$ is the above described operator.

Theorem. If $g(x, r)$ satisfies (a) and (b) and $G(x, r)$ is its primitive with respect to $r$ then, if $V$ is any closed subspace of $W_{0}^{1, p}(\Omega)$ and $K \subset V$ is a closed, convex subset of $V$ with $0 \in K$ and $f \in V^{\prime}$ then, there is a unique $u \in K$ such that $g(x, u)$ is in $L^{1}(\Omega), g(x, u) u$ is in $L^{1}(\Omega)$ and $\int G(x, u) d x<\infty$. Moreover, $u$, satisfies both inequalities:

(i) for each $v \in K \cap L^{\infty}(\Omega)$,

$(A(u)+g(x, u)-f, v-u) \geq 0$

(ii) for each $v \in K$, $\int G(x, v) d x-\int G(x, u) d x+(A(u)-f, v-u) \geq 0$. 
Proof: We know, from [3] that for each positive, integer $n$, there is a solution $u_{n}$, in $K$ of the variational inequality:

$$
\left(A\left(u_{n}\right)+g_{n}\left(x, u_{n}\right)-f, v-u_{n}\right) \geq 0, \quad(v \in K) .
$$

Since $A$ is coercive and $0 \in K$,

$$
\left(A\left(u_{n}\right)+g_{n}\left(x, u_{n}\right)-f, u_{n}\right) \leq 0 .
$$

Therefore,

$$
\alpha\left\|u_{n}\right\|^{p} \leq\left(A\left(u_{n}\right), u_{n}\right) \leq\left(A\left(u_{n}\right)+g_{n}\left(x, u_{n}\right), u_{n}\right) \leq\left(f, u_{n}\right) . \quad *
$$

with $\alpha \in \mathbf{R}$.

We will show that, if

$$
u_{n} \rightarrow u, \quad \text { weakly in } V,
$$

$u$ is a solution of the problem in the theorem and that

$$
w=A(u) .
$$

From $(*)$, we have that

$$
\int_{\Omega} g_{n}\left(x, u_{n}\right) u_{n} d x
$$

is uniformly bounded, for all $n$.

The sequence $\left\{g_{n}\left(x, u_{n}\right)\right\} \_n$ is equiuniformly integrable on $\Omega$.

For each $R$, positive, integer,

$R\left|g_{n}\left(x, u_{n}\right)\right| \leq u_{n} g_{n}\left(x, u_{n}\right)+R\{g(x, R)+|g(x,-R)|\}$, $* *$ since $g(x, \cdot)$ is non-decreasing.

Let $\varepsilon>0$ and $B \subset \Omega$, measurable. We have

$$
\int_{B}\left|g_{n}\left(x, u_{n}\right)\right| d x \leq \frac{1}{R} \int_{B} u_{n} g_{n}\left(x, u_{n}\right) d x+\int_{B} g(x, R) d x+|g(x,-R)|
$$

and this may be taken less than $\varepsilon$ for all $n$ if $\mu(B)$ is sufficiently small, as far as $g(\cdot, r) \in L^{1}(\Omega)$. 
From inequality $(* *)$, with $N \subset \Omega$,

$\int_{N}\left|g_{n}\left(x, u_{n}\right)\right| d x \leq \frac{1}{R} \int_{N} u_{n} g_{n}\left(x, u_{n}\right) d x+\int_{N}(g(x, R) d x+|g(x,-R)|) d x$.

Since $\int_{N} u_{n} g_{n}\left(x, u_{n}\right) d x \leq M_{2}$, independently of $n$, there exists $B_{\varepsilon} \subset \Omega$ measurable with $\mu\left(B_{\varepsilon}\right)<\infty$, such that

$$
\int_{\Omega-B_{\varepsilon}}\left|g_{n}\left(x, u_{n}\right)\right| d x \leq \varepsilon, \quad \text { for all } \quad n \in \mathbf{N} .
$$

Moreover, since $\left\|u_{n}\right\| \leq C$ by the Sobolev immersion theorems, we may obtain $\left(u_{n}\right)$ a subsequence of $\left(u_{n}\right)$ such that

$$
u_{n} \rightarrow u, \quad \text { a.e. in } \Omega \text {. }
$$

Therefore

$$
g_{n}\left(x, u_{n}\right) \rightarrow g(x, u), \quad \text { a.e., in } \Omega .
$$

By the convergence theorem of Vitali, $g(x, u)$ is in $L^{1}(\Omega)$, and

$$
g_{n}\left(x, u_{n}\right) \rightarrow g(x, u)
$$

strongly in $L^{1}(\Omega)$. Using Fatou's lemma, $g(x, u) u$ is in $L^{1}(\Omega)$.

For each $n \in \mathbf{N}$, let us define

$$
G_{n}(x, r)=\int_{0}^{r} g_{n}(x, s) d s .
$$

For each $r$ and $s$,

$$
\begin{array}{r}
G_{n}(x, r)-G_{n}(x, s)=G_{n}^{\prime}(x, \xi)(r-s)=g_{n}(x, \xi)(r-s) \\
\geq g_{n}(x, s)(r-s), r \leq \xi \leq s .
\end{array}
$$

Let $v \in K$ be arbitrary. We have:

$$
G_{n}(x, v)-G_{n}\left(x, u_{n}\right) \geq g_{n}\left(x, u_{n}\right)\left(v-u_{n}\right) .
$$

Integrating over $\Omega$, we obtain:

$$
\int G_{n}(x, v)-G_{n}\left(x, u_{n}\right) \geq \int g_{n}\left(x, u_{n}\right)\left(v-u_{n}\right) \geq\left(f-A\left(u_{n}\right), v-u_{n}\right) .
$$


If $v$ is such that $\int G(x, v)<\infty$ then

$$
\left|G_{n}(x, v)\right| \leq|G(x, v)|
$$

what implies that

$$
\int G_{n}(x, v) \rightarrow \int G(x, v) .
$$

We also have

$$
G_{n}\left(x, u_{n}\right) \rightarrow G(x, u) \quad \text { a.e. in } \Omega .
$$

Moreover,

$$
G(x, u(x))=\int_{0}^{u(x)} g(x, s) d s \leq g(x, u(x)) u(x),
$$

and since $g(x, u) u \in L^{1}(\Omega)$,

$$
\int G(x, u) d x<\infty .
$$

We obtain,

$$
\int G(x, v)-\int G(x, u) \geq \lim \sup \left(A\left(u_{n}\right)-f, u_{n}-V\right),
$$

for each $v \in K$, such that

$$
\int G(x, v) d x<\infty .
$$

Letting, $v=u$, we have

$$
0 \geq \lim \sup \left(A\left(u_{n}\right)-f, u_{n}-u\right)=\lim \sup \left(A\left(u_{n}\right), u_{n}-u\right) .
$$

Since $A$ is pseudo-monotonic from $V$ to $V^{\prime}, w=A(u)$ that is,

$$
A\left(u_{n}\right) \quad \text { converges weakly to } A(u)
$$

in $V^{\prime}$, and

$$
\left(A\left(u_{n}\right), u_{n}\right) \rightarrow(A(u), u) .
$$


Therefore, for each $v \in K$ with

$$
\int G(x, v) d x<\infty
$$

we have:

$$
\int G(x, v)-\int G(x, u) \geq(A(u)-f, u-v),
$$

which is part our theorem.

Let now, $v \in K \cap L^{\infty}(\Omega)$.

We have,

$$
\int g_{n}\left(x, u_{n}\right)\left(v-u_{n}\right) \geq\left(A\left(u_{n}\right)-f, u_{n}-v\right) .
$$

By the lemma of Fatou, we have, since $v \in L^{\infty}(\Omega) \cap K$ :

$\liminf g_{n}\left(x, u_{n}\right)\left(v-u_{n}\right) \geq \liminf \left(A\left(u_{n}\right)-f, u_{n}-v\right)=(A(u)-f, u-v)$.

Therefore

$$
\int g(x, u)(v-u) \geq(A(u)-f, u-v)
$$

or

$$
(A(u)+g(x, u)-f, v-u) \geq 0
$$

what is other part of our theorem.

\section{Unicity}

Let $u_{1}$ and $u_{2}$ be two solutions of our problem, for a given $f \in V^{\prime}$.

Then,

$$
\int G(x, v)-\int G\left(x, u_{1}\right) \geq\left(A\left(u_{1}\right)-f, u_{1}-v\right)
$$

and

$$
\int G(x, v)-\int G\left(x, u_{2}\right) \geq\left(A\left(u_{2}\right)-f, u_{2}-v\right) .
$$

$G(x, r)$ is convex in $r$. Hence if we put

$$
v=\frac{1}{2}\left(u_{1}+u_{2}\right)
$$


$v$ is a permissible element, and

$$
u_{1}-v=\frac{1}{2}\left(u_{1}-u_{2}\right)=-\left(u_{2}-v\right) .
$$

Hence,

$$
\begin{aligned}
& \int G(x, v)-\int G\left(x, u_{1}\right) \geq \frac{1}{2}\left(A u_{1}-f, u_{1}-u_{2}\right) \\
& \int G(x, v)-\int G\left(x, u_{2}\right) \geq \frac{1}{2}\left(A u_{2}-f, u_{2}-u_{1}\right) .
\end{aligned}
$$

Adding the inequalities, we obtain:

$\frac{1}{2}\left(A\left(u_{1}\right)-A\left(u_{2}\right), u_{1}-u_{2}\right)+\int G\left(x, u_{1}\right)+\int G\left(x, u_{2}\right)-2 \int G(x, v) \leq 0$.

Therefore

$0 \leq\left(A u_{1}-A u_{2}, u_{1}-u_{2}\right)+2\left[2 \int \frac{G\left(x, u_{1}\right)+G\left(x, u_{2}\right)}{2}-G\left(x, \frac{u_{1}+u_{2}}{2}\right)\right]$

$\leq 0$.

$G$ is convex and therefore the second term is zero. Hence,

$$
\begin{gathered}
\left(A u_{1}-A u_{2}, u_{1}-u_{2}\right)=0 \\
\sum_{i=1}^{n} \int_{\Omega}\left(\left|\frac{\partial u}{\partial x_{i}}\right|^{p-2} \frac{\partial u_{1}}{\partial x_{i}}-\left|\frac{\partial u_{2}}{\partial x_{i}}\right|^{p-2} \frac{\partial u_{2}}{\partial x_{i}}\right)\left(\frac{\partial u_{1}}{\partial x_{i}}-\frac{\partial u_{2}}{\partial x_{i}}\right) d x=0 .
\end{gathered}
$$

The function

$$
\lambda \rightarrow|\lambda|^{p-2} \lambda
$$

is monotone. Therefore, for each $i$,

$$
\left(\left|\frac{\partial u_{1}}{\partial x_{i}}\right|^{p-2} \frac{\partial u_{1}}{\partial x_{i}}-\left|\frac{\partial u_{2}}{\partial x_{i}}\right|^{p-2} \frac{\partial u_{2}}{\partial x_{i}}\right)\left(\frac{\partial u_{1}}{\partial x_{i}}-\frac{\partial u_{2}}{\partial x_{i}}\right)=0
$$

for almost all $x \in \Omega$.

By the same reason, $\frac{\partial u_{1}}{\partial x_{i}}=\frac{\partial u_{2}}{\partial x_{i}}$, for each $i$.

But $u_{1}-u_{2}=0$, on $\Gamma$, since $u_{1}-u_{2} \in W_{0}^{1, p}(\Omega)$. Therefore,

$$
u_{1}=u_{2}
$$




\section{References}

[1] Brézis, H., Integrales convexes dans les espaces de Sobolev, Israel J. Math., 13, pp. 9-23, (1972).

[2] Brézis, H. and Browder, F., Strongly Nonlinear Elliptic Boundary Value Problems, Ann. Sc. Norm. Sup. di Pisa, pp. 587-598, (1978).

[3] Lions, J.L., Quelques Méthodes de Résolution des Problèmes aux Limites non Linéaires, Ed. Dunod, Paris, (1969).

[4] Natanson, I., Theory of Functions of a Real Variable, Ed. Frederick Ungar, N.Y., (1955).

Received : May, 1999.

\section{A. Wanderley}

Instituto de Matemática e Estatística

Universidade do Estado do Río de Janeiro

Rua Sâo Francisco Xavier, 524

Río de Janeiro

RJ 20550 - 013

Brasil

and

\section{N. Riberiro}

Instituto de Matemática

Centro de Tecnología, Bloco C

Universidade Federal do Río de Janeiro

Río de Janeiro

RJ 21945 - 9701

Brasil 